\title{
Increased psychotropic drug consumption by children in the Netherlands during 1995-2001 is caused by increased use of methylphenidate by boys
}

Received: 13 November 2003/ Accepted: 16 March 2004 / Published online: 28 May 2004

(C) Springer-Verlag 2004

\begin{abstract}
Objective: The aim of the study was to determine the changes in consumption of psychotropic drugs by children aged less than 18 years during the years 1995 to 2001 in the Netherlands.

Methods: The year prevalence of antipsychotics, benzodiazepines, antidepressants and psychostimulants for boys and girls under 18 years was determined using electronic pharmacy dispensing records obtained from the PHARMO database.

Results: The overall prevalence of psychotropic drugs increased from 11.1 per 1000 in 1995 to 22.9 per 1000 in 2001. This increase could almost completely be attributed to the increase in the use of psychostimulants, i.e. methylphenidate, which increased from 1.7 per 1000 children in 1995 to 10.0 per 1000 in 2001 . For the other psychotropic drugs, no or only a small increase was seen. For both boys and girls, the use of psychostimulants was highest in the age group of 5-14 years.

Conclusion: During the years 1995-2001, the consumption of psychotropic drugs by children in the Netherlands has more than doubled. This increase could largely be attributed to an increased use of the psychostimulant methylphenidate by boys of the age 5-14 years.
\end{abstract}

Keywords Pharmacoepidemiology · Children · Psychotropic drugs

\section{Introduction}

Several investigators have shown that in the United States the use of psychotropic drugs by children has

J. G. Hugtenburg $(\bowtie) \cdot$ E. R. Heerdink · A. C. G. Egberts Utrecht Institute for Pharmaceutical Sciences,

Department of Pharmacoepidemiology and Pharmacotherapy, Utrecht University, PO Box 80082, 3508 TB Utrecht,

The Netherlands

E-mail: J.G.Hugtenburg@pharm.uu.nl

Tel.: + 31-030-2537324

Fax: + 31-020-2539166 sharply increased during recent years [1, 2, 3, 4]. Also, in European countries psychiatric drug prescribing for children seems to have increased, though less clearly than in the United States [5]. Public concern regarding this phenomenon, especially regarding possible longterm adverse effects and future drug abuse has been raised. We therefore evaluated the changes in the use of psychotropic drugs in children less than 18 years in the Netherlands during the period 1995-2001.

\section{Materials, methods and subjects studied}

The year prevalences of use of antipsychotics (ATC-code N05A), benzodiazepines (ATC-code N05B, N05C), antidepressants (ATC-code N06A) and psychostimulants (ATC-code N06B) were determined using the PHARMO record linkage system. This database currently contains prescription data from retail pharmacies of a defined population of around 1 million residents of the Netherlands.

Year prevalences were calculated overall as well as stratified according to gender and different age categories for each calendar year from 1995 to 2001 . The numerator was the number of children that received at least one prescription for the specified drugs. The nominator was the mean number of children aged less than 18 years of the defined population in the specified calendar year.

\section{Results}

Year prevalences of the use of antipsychotics, anxiolytics, benzodiazepines, antidepressants and psychostimulants for boys and girls are shown in Fig. 1. Overall, the use of psychotropic drugs increased fourfold from 11.1 per 1000 children in 1995 to 22.9 per 1000 children in 2001. This increase could largely be attributed to the increase in the use of psychostimulants, i.e. methylphenidate, which increased from 1.7 per 1000 children in 1995 to 10.0 per 1000 in 2001 . For other drugs, no or only a small increase was seen. 
Fig. 1 Prevalence of psychotropic drug use in children (1995-2001). Increase of psychotropic drug consumption by children in the Netherlands is caused by increased use of methylphenidate by boys
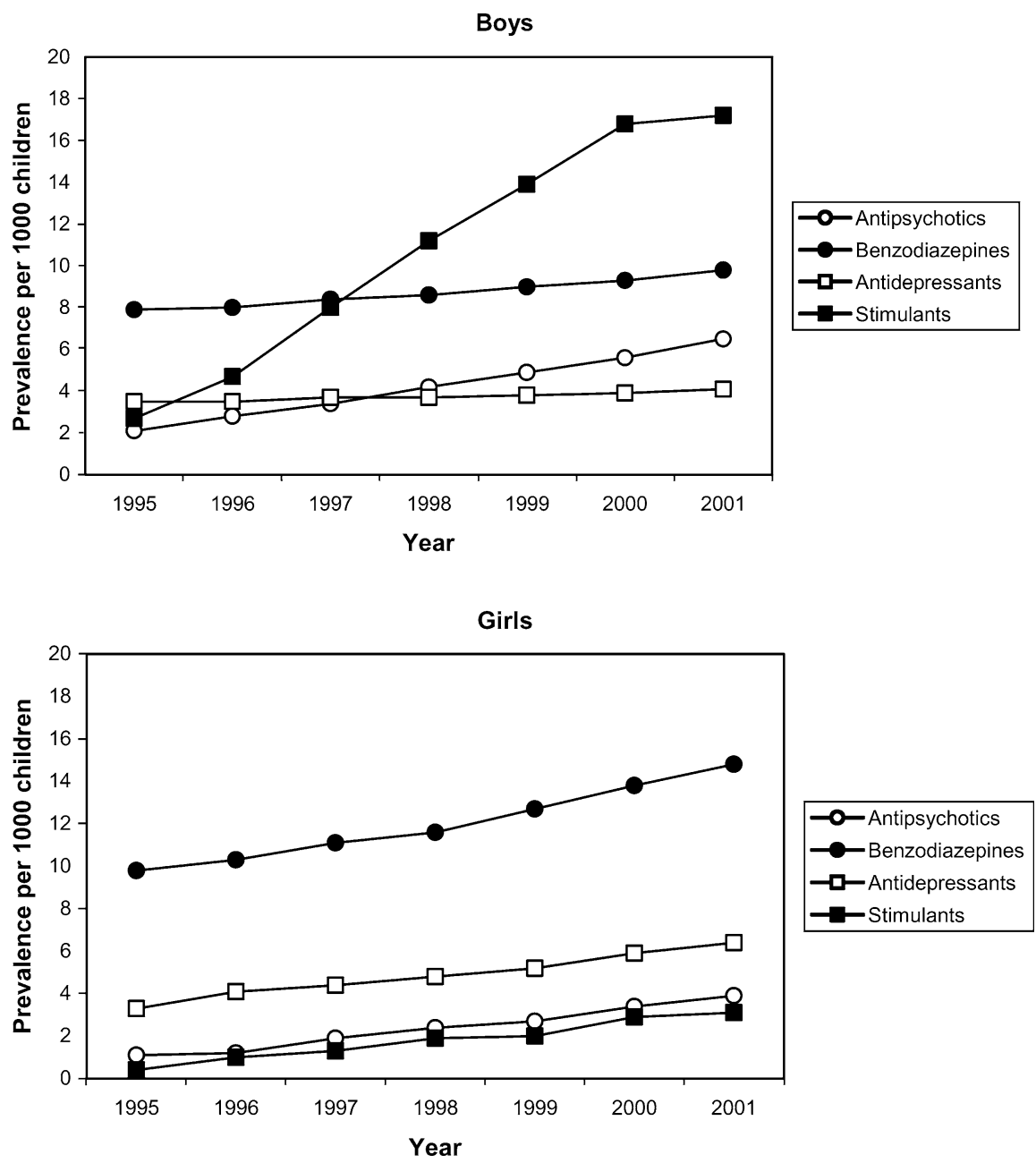

For both boys and girls, the use of psychostimulants was highest in the age groups 5-9 years and 10-14 years. Boys between 5 years and 14 years of age showed a much higher prevalence than girls (in 2001, 26.2 per 1000 boys versus 3.8 per 1000 girls).

\section{Discussion and conclusions}

Our main finding was that the consumption of psychotropic drugs by children in the Netherlands has more than doubled during the years 1995-2001. This increase is mainly caused by an increased use of psychostimulants by boys of the age 5-14 years.

In a drug utilisation study performed in the Northern part of the Netherlands a sharp increase of the use of psychostimulants was also found [5].

In 1995, the use of psychostimulants in the Netherlands was five- to tenfold lower than in the United States. In 2001, the use of psychostimulants in the Netherlands was about twofold lower than the use in the United States in 1995.

Zito and co-workers have studied the prevalence of methylphenidate prescribing in the United States using various insurance claim databases [3]. The prevalence in children aged 5-14 years varied from 10.3 to 21.4 per 1000 per year [3]. For children aged 5-18 years, the prevalence increased from 11.5 per 1000 in 1990 to 33.6 per 1000 in $1995[2,4]$. In a study among public school children, a prevalence of 24 per 1000 was found [1]. In Great Britain, the prevalence of psychostimulants was much lower, with 0.3 per 1000 in school children in 1995 [6]. Although differences in study design and years studied may play a role, the differences - being two- to fivefold - are more likely to be explained in terms of differences in life-style, public knowledge of attention deficit hyperactivity disorder and medical practice systems. In addition, resistance against prescribing drugs for children with psychiatric disorders may be lower in the United States than in European countries.

\section{References}

1. Safer DJ, Malaver M (2000) Stimulant treatment in Maryland Public School. Pediatrics 106:533-539

2. Robison LM, Sclar DA, Traer TL, Galin RS (1999) National trends in the prevalence of attention-deficit/hyperactivity disorder and the prescribing of methylphenidate among school-age children: 1990-1995. Clin Pediatrics 38:209-217 
3. Zito JM, Safer DJ, Riddle MA, Johnson RE, Speedie SM, Fox M (1998) Prevalence variations in psychotropic treatment of children. J Am Child Adolesc Psychopharmacol 8:99-105

4. Safer DJ, Zito JM, Fine EM (1996) Increased methylphenidate usage for attention deficit disorder in the 1990s. Pediatrics 98:1084-1088
5. Schirm E, Tobi H, Zito JM, De Jong-van den Berg LTW (2001) Psychotropic medication in children: a study from the Netherlands. Pediatrics 108:e25-e29

6. Kewley GD (1998) Attention deficit hyperactivity disorder is underdiagnosed and undertreated in Britain. BMJ 316:15941596 